\title{
Hamstring tendon anterior cruciate ligament reconstruction: does gracilis tendon harvest matter?
}

\author{
Björn Barenius • Webster Kate Webster • \\ Jodie McClelland $\cdot$ Julian Feller
}

Received: 24 July 2012 / Accepted: 27 September 2012 / Published online: 11 October 2012

(C) Springer-Verlag Berlin Heidelberg 2012

\begin{abstract}
Purpose Hamstring tendon grafts are a popular choice for anterior cruciate ligament (ACL) reconstruction. Typical morbidity reported after hamstring tendon harvest is muscle weakness in flexion, and whilst still a matter for debate, this morbidity may be greater if the gracilis tendon is harvested in addition to the semitendinosus.

This study sought to comprehensively compare the outcome of ACL reconstructions in which the semitendinosus was harvested alone (ST group) or with the gracilis (ST/G group).

Methods Twenty patients (ten ST, ten ST/G) were assessed with a variety of measures that included subjective scores, function, strength and tibial rotation as measured by gait analysis during a pivoting task.

Results Results showed that the graft diameter was significantly larger in the ST group, but there were no other differences between the groups for any other outcome measure. Conclusion Harvesting the gracilis in addition to semitendinosus does not appear to affect either the outcome or graft-related morbidity after hamstring ACL reconstruction.
\end{abstract}

\section{Introduction}

Anterior cruciate ligament (ACL) reconstruction surgery aims primarily to restore knee joint stability so that patients

\footnotetext{
B. Barenius $(\triangle)$

Department of Clinical Science and Education, Karolinska Institutet, Södersjukhuset,

Stockholm, Sweden

e-mail: bjorn.barenius@sodersjukhuset.se
}

B. Barenius $\cdot$ W. K. Webster $\cdot$ J. McClelland $\cdot$ J. Feller

Musculoskeletal Research Centre, Faculty of Health Sciences, La

Trobe University,

Melbourne, Australia can return to pivoting sports with a low risk for graft ruptures and graft-related morbidity. Previous studies of the biomechanical properties of bone patellar tendon bone (PT) and hamstring tendons have found the PT graft to be stiffer and stronger [1]. Patellar tendon ACL reconstruction has been regarded by many to be the so-called 'gold standard' [2]. However, persistent morbidity after PT graft harvest [3] has led to the increased use of a four-strand semitendinosus and gracilis graft, which has comparable laxity and graft rupture rates $[4,5]$.

Typical morbidity after hamstring graft harvest is decreased strength in knee flexion and tibial rotation $[6,7]$. This has led to a debate regarding the harvest of the gracilis tendon. Some authors recommend harvesting only the semitendinosus tendon to minimise morbidity $[8,9]$. Others have not found any significant differences in hamstring strength during flexion when a harvest of both the semitendinosus and gracilis tendons $(\mathrm{ST} / \mathrm{G})$ was compared with a harvest of only the semitendinosus tendon (ST) $[10,11]$. In theory, the four-strand ST graft could provide better stability in translation and rotation due to its larger diameter and greater stiffness compared to the ST/G [1]. The main drawback with the ST graft is that the tendon length might not be adequate for fixation of the tendon in the bone tunnels [9].

The evaluation of tibial rotation with gait analysis has shown comparable results after $\mathrm{PT}$ and $\mathrm{ST} / \mathrm{G}$ reconstructions [12-15], but to our knowledge the ST graft reconstruction has not been evaluated with gait analysis with regard to knee rotation. Adding a gait analysis protocol to other more traditional outcome measures may provide further information to evaluate graft choice [14]. This study therefore aimed to comprehensively compare ACL reconstructions using a traditional hamstring tendon graft $(\mathrm{ST} / \mathrm{G})$ with reconstructions using a four-strand semitendinosus tendon graft (ST), using subjective outcome scores, measures of knee laxity, function, strength and gait analysis. 


\section{Methods}

Participants

The Human Ethics Committee of La Trobe University approved this study. Patients were informed of the nature of the experiment and gave written consent to participate before testing.

Twenty patients were assessed at a mean of 36 months after reconstruction surgery (range 27-45 months). Ten patients had undergone primary ACL reconstruction using a conventional hamstring autograft with doubled semitendinosus and gracilis tendons (ST/G), and ten patients had an ACL reconstruction with a four-strand semitendinosus tendon autograft (ST). Exclusion criteria from the study were: previous anterior cruciate ligament damage to either knee, chondral disruption greater than Noyes grade IIA [16], meniscus pathology treated by repair and current symptoms from the hip or ankle.

A single experienced knee surgeon performed the ACL reconstructions arthroscopically. The surgical technique was the same for both groups. The tendons were harvested through an oblique 3- to 4-cm incision. The semitendinosus tendon was always harvested first and the semitendinosus tendon length was measured. A minimum length of $26 \mathrm{~cm}$ for the semitendinosus tendon was required to perform a four-strand semitendinosus only reconstruction. The tendon graft was attached with a doubled $3 \mathrm{~mm}$ Dacron tape to an Endobutton (Smith and Nephew Endoscopy, Mansfield, MA, USA). The femoral tunnel was drilled using a transtibial technique, but with the starting point of the femoral tunnel identified prior to tibial tunnel preparation, and the starting point of the tibial tunnel more medial and proximal than for a "traditional" transtibial technique. The surgeon aimed to start the femoral tunnel at the " 10 o'clock" orientation on the lateral wall of the intercondylar notch for a right knee, as visualised with the knee at $80^{\circ}$ flexion, approximating the centre point of the femoral footprint of the ACL. The distal end of the graft was fixed with interference screw with the knee in $70^{\circ}$ of flexion and $67 \mathrm{~N}$ of traction applied to the graft. Patients were discharged from hospital on the first postoperative day. All patients underwent the same rehabilitation protocol, which has been previously described [14]. This protocol encouraged immediate full knee extension and the restoration of quadriceps function as soon as possible. Weight bearing was allowed on an astolerated basis from the first postoperative day.

\section{Outcome measures}

All data were collected in a single two-hour session. Demographic information was collected and the patients completed the subjective IKDC 2000 form [17]. They were asked if they had returned to sport activities and at what level, and also whether they had experienced hamstring pain or hamstring problems after their reconstructive surgery. Graft size was defined as the femoral tunnel size from the surgical protocol. A single examiner measured knee range of motion with a goniometer, and performed a Lachman test and pivot shift test. The pivot shift was graded as nearly normal if there was a "glide" or if there was any discernible difference in the pivot shift noted between the healthy contralateral limb and the operated limb, i.e. a difference that was "less" than a glide. Instrumented anterior laxity was measured with the KT-1,000 arthrometer (MEDmetric, San Diego, CA) at maximal manual force and a difference between the reconstructed and the healthy limb was calculated.

A test of hamstring strength was performed in a seated position with a Biodex dynamometer in a manner previously described (Biodex Medical Systems, Shirley, NY) [10]. A five minute warm-up on a stationary exercise bicycle preceded the testing. The healthy limb was tested first in all patients. Isokinetic and isometric hamstring strength was measured in Newton-metres $(\mathrm{Nm})$ and compared to the contralateral healthy limb. Torque was measured at 20 and $90^{\circ}$ of flexion for the isokinetic test and at $90^{\circ}$ of flexion for the isometric test. Active knee flexion angle according to Nakamura et al. was also measured [6].

For measuring tibial rotation, length measurements of the pelvis and lower limbs were obtained for each patient and reflective markers were attached to the lower limbs using the standard Plug-in-Gait marker set [18, 19]. Data were captured using an eight camera three-dimensional motion analysis system with a sampling rate set to $100 \mathrm{~Hz}$ (Vicon MX system, Oxford Metrics Ltd, UK). The system was calibrated according to the manufacturer's instructions prior to each data collection session. To obtain a reference point for the markers, a static trial was obtained with the patient in quiet standing. For this trial, a knee alignment device was used to determine the centre of the knee joint as previously described [14]. After a series of warm-up trials of walking, rotational laxity of the knee was assessed during a stair descent and pivot activity. This task has been previously described [18] and was essentially the same as the procedure used by Georgoulis et al. [13]. Patients were required to descend two steps to the floor with alternating feet on each step. Immediately after foot contact with the ground, the patients pivoted (externally rotated on the stance limb) on the landing leg $90^{\circ}$ (to the right for the right leg and vice versa) and walked away from the staircase. All patients were instructed to descend and pivot at a self-selected pace as quickly and safely as they could. A minimum of six trials was conducted for each limb with the healthy limb tested first. Vicon Plug-in-Gait (Oxford Metrics) biomechanical modelling software was used to process and output the data as kinematic profiles as previously described [18]. The pivoting period was identified from 
initial foot contact with the ground (ipsilateral limb) until foot contact with the ground of the contralateral limb. Therefore, only data from the stance phases of the 'pivoting' limb was analysed. Tibial rotation profiles were plotted and the maximum and minimum angular displacements identified from each of the six trials for each subject. The difference between these two points was calculated to represent the range of tibial rotation as previously described, and this was the kinematic variable of interest $[12,20]$.

Statistical analysis

SPSS $^{\text {TM }}$ version 20 for Macintosh was used for statistical analysis. Nominal variables were tested by the chi-square test or Fisher's exact test. Ordinal variables and nonnormally distributed interval and ratio scale variables were evaluated by the Mann-Whitney U-test. Student's $t$-test was used for normally distributed interval and ratio scale variables in independent groups. Paired samples $t$-test was used for related samples. The tests were two-sided. The results were considered significant at $p<0.05$.

\section{Results}

Demographic data for the two graft groups are presented in Table 1. The patients in the ST group were on average six centimetres taller than the ST/G patients (Table 1); the groups were otherwise well matched. Associated injuries are listed in Table 2. The ST tendon grafts were significantly thicker with a mean diameter of $8.7 \mathrm{~mm}$ (SD 1) compared to the ST/G grafts with a mean diameter of $7.5 \mathrm{~mm}(\mathrm{SD} 1)(p=0.01)$. There was no difference between the ST and ST/G groups for the subjective IKDC overall score or the specific questions regarding function prior to injury or current function (Table 3). There was also no between-group difference in active range of motion (ROM) (Table 3). Both groups showed a loss of active ROM after the reconstructive surgery. When averaged across all patients the mean deficit of the operated limb was $3.5^{\circ}$ compared to the non-injured healthy limb $(p=0.02)$ (Table 3).

None of the patients described symptoms of instability after their reconstructive surgery. The Lachman and pivot shift tests were normal or nearly normal for all patients with no significant differences between the graft groups (Table 3 ).

In both groups nine out of ten patients had returned to sports. Five patients in the ST group and six in the ST/G group reported that they had experienced an episode of hamstring pain after the reconstruction. One patient in each group reported hamstring pain of a persistent nature. The patient in the ST/G group reported cramps in inner range flexion, and the patient in the ST group reported frequent hamstrings strains during Australian Rules football.

Both graft groups were similar regarding hamstring strength, but both showed a strength deficit in the order of $15-20 \%$ in the reconstructed limb compared to the healthy limb in $90^{\circ}$ of flexion (Table 3 ).

Results for tibial rotation are presented in Fig. 1. The range of rotation of the reconstructed limb was similar between the groups. In both the ST and ST/G groups, there were no significant differences between the reconstructed and the non-injured healthy limbs.

\section{Discussion}

This study showed that harvesting the gracilis tendon did not have a significant negative influence on the outcome or graft-related morbidity after an ACL reconstruction.
Table 1 Demographic results. Semitendinosus and gracilis tendon group (ST/G) and semitendinosus tendon group (ST)

\begin{tabular}{llll}
\hline Variable & $\mathrm{ST} / \mathrm{G}$ & $\mathrm{ST}$ & Sign. \\
\hline Gender (female), $n(\%)$ & $2(20)$ & $2(20)$ & 1 \\
Injured limb (right), $n(\%)$ & $5(50)$ & $6(60)$ & 0.7 \\
Height (cm), mean (SD) & $173(5)$ & $179(6)$ & 0.03 \\
Limb length, mean (SD) & $90(5)$ & $95(5)$ & 0.08 \\
Weight (kg), mean (SD) & $78(12)$ & $83(13)$ & 0.4 \\
Age at injury (years), mean (SD) & $26(9)$ & $26(7)$ & 0.9 \\
Time between injury and reconstruction (days), mean (SD) & $84(82)$ & $91(62)$ & 0.8 \\
Time between reconstruction and follow up (months), mean (SD) & $37(6)$ & $36(4)$ & 0.6 \\
Sport at injury, $n$ & & & 0.8 \\
Soccer & 3 & 2 & \\
Australian rules football & 3 & 2 & \\
Netball & 1 & 2 & \\
Basketball & 1 & 2 & \\
Other, tennis, martial arts, lacrosse & 2 & 2 & \\
\hline
\end{tabular}


Table 2 List of additional injuries and treatment. Semitendinosus and gracilis tendon group (ST/G) and semitendinosus tendon group (ST)

\begin{tabular}{lll}
\hline Graft type & Additional injury & Treatment \\
\hline $5 \mathrm{ST} / \mathrm{G}$ & No additional injury & \\
ST/G & Lateral meniscus tear & No treatment \\
ST/G & Lateral meniscus tear & Partial resection \\
ST/G & Cartilage injury patella and patellar grove Noyes grade 2a & Shaving \\
ST/G & Lateral meniscus tear & No treatment \\
ST/G & Cartilage injury medial femoral condyle Noyes grade 2a & No treatment \\
$6 \mathrm{ST}$ & No additional injury & \\
ST & MCL tear, stable & No treatment \\
ST & Lateral meniscus tear & No treatment \\
ST & Cartilage injury medial femoral condyle Noyes grade 2a & Shaving \\
ST & Lateral meniscus tear & No treatment \\
\hline
\end{tabular}

A novel aspect of this study was the use of kinematic gait analysis of a dynamic task to compare the two graft types. Residual rotational laxity has been reported after ACL reconstructions with $\mathrm{PT}$ and $\mathrm{ST} / \mathrm{G}$ grafts $[13,16]$. Contrary to these reports, we found that in our patients, the range of tibial rotation in the ACL reconstructed knee was similar to the contralateral knee. This is consistent with previously published results from our laboratory using a different group of patients [20]. That the range of tibial rotation was also similar between the ST and the ST/G groups suggests that the residual rotational laxity reported in other studies might be an effect of something other than graft type, such as tunnel placement [1].

We found a flexion strength deficit after hamstring harvest but no difference in knee flexion strength between the graft groups when the ACL reconstructed limb was compared to the healthy non injured limb, which is consistent with previous publications $[10,11]$. The replication of these findings indicates that the additional harvesting of the gracilis graft does not result in greater hamstring strength deficits.

Therefore, the decision to use either a semitendinosus and gracilis or four-strand semitendinosus graft should
Table 3 Comparison of outcome measures between the graft groups. Semitendinosus and gracilis tendon group (ST/G) and semitendinosus tendon group (ST) $n$ for all comparisons $9-10$ except active flexion angle ST/G group where $n=7$

\begin{tabular}{|c|c|c|c|}
\hline Outcome measures & $\begin{array}{l}\mathrm{ST} / \mathrm{G} \\
\text { Mean }(\mathrm{SD}) / n\end{array}$ & $\begin{array}{l}\mathrm{ST} \\
\text { Mean (SD) } / n\end{array}$ & Sign. \\
\hline Graft diameter, $(\mathrm{mm})$ & $7.5(1)$ & $8.7(1)$ & 0.01 \\
\hline IKDC subjective, $(0-100)$ & $94.5(4.0)$ & $94.1(3.7)$ & 0.8 \\
\hline IKDC function prior to injury, $(0-11)$ & $11(0)$ & $10.8(0.7)$ & 0.3 \\
\hline IKDC Current function at follow up, $(0-11)$ & $9.8(1.0)$ & $9.4(0.9)$ & 0.5 \\
\hline Loss of active ROM compared to healthy limb, (degrees) & $6(7)$ & $1(5)$ & 0.1 \\
\hline Active flexion angle compared to healthy limb, (degrees) & $7(11)$ & $4(5)$ & 0.4 \\
\hline KT1000 side to side difference, $(\mathrm{mm})$ & $1.3(3.5)$ & $1.8(2.5)$ & 0.7 \\
\hline Lachman & & & 0.4 \\
\hline Grade 0 & 3 & 5 & \\
\hline Grade 1 & 6 & 4 & \\
\hline Not performed & 1 & 1 & \\
\hline Pivot shift & & & 0.3 \\
\hline Absent & 8 & 5 & \\
\hline Nearly normal & 1 & 4 & \\
\hline Not performed & 1 & 1 & \\
\hline Difference in isokinetic muscle torque at $20^{\circ}$ flexion, $60 \% \mathrm{~s}(\mathrm{Nm})$ & $-1.4(16)$ & $-1.1(21)$ & 1.0 \\
\hline Difference in isokinetic muscle torque at $90^{\circ}$ flexion, $60 \% \mathrm{~s}(\mathrm{Nm})$ & $22.0(36)$ & $14.2(30)$ & 0.6 \\
\hline Isokinetic strength as percent of healthy limb at $90^{\circ}, 60 \% \mathrm{~s}(\%)$ & $78.0(36)$ & $85.8(30)$ & 0.6 \\
\hline Difference in isometric muscle torque at $90^{\circ}(\mathrm{Nm})$ & $15.8(20)$ & $17.2(29)$ & 0.9 \\
\hline Isometric strength as percent of healthy limb at $90^{\circ}(\%)$ & $82.6(27)$ & $82.7(36)$ & 1.0 \\
\hline
\end{tabular}


Fig. 1 Range (SD) of tibial rotation measured by gait analysis in degrees. White columns healthy non-injured limb, grey columns ACL reconstructed limb, ST4 semitendinosus tendon group, $S T / G$ semitendinosus and gracilis tendon group, All combined data for the whole cohort

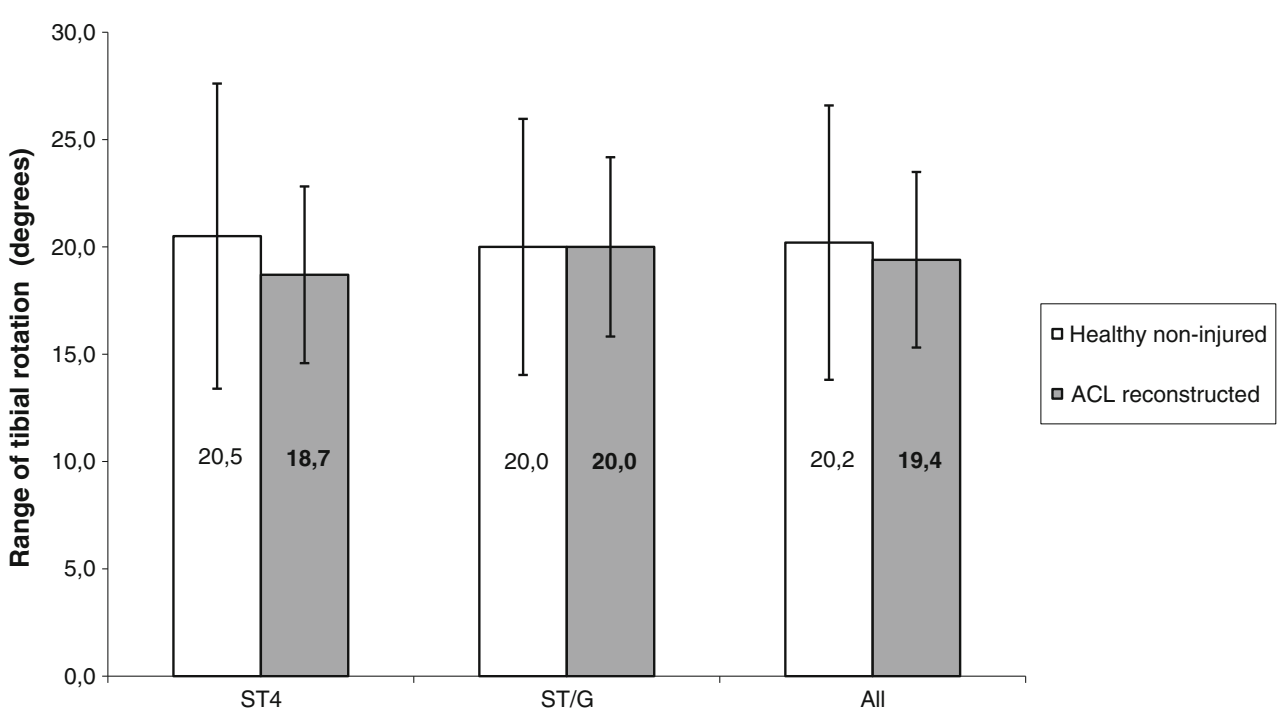

include consideration of issues other than range of knee rotation and harvest-related morbidity such as hamstring strength. Prodromos has listed some concerns relevant to the decisions a surgeon has to make regarding the hamstring graft construct, specifically, graft length in the tunnels, preferred fixation method, the need for gracilis harvest or not and graft strength [21]. Other authors have proposed that a semitendinosus tendon length of at least $28 \mathrm{~cm}$ is required to have enough graft length in the tunnels to do a four-strand semitendinosus ACL reconstruction [9]. Consistent with this, we found a difference in body height between the groups, which probably reflects our requirement for a semitendinosus tendon length of $26 \mathrm{~cm}$ to perform a four-strand semitendinosus ACL reconstruction. The difference in height between the graft groups might also in part explain the difference in graft diameter [22]. However there are reports suggesting that a shorter graft length inside the tunnels does not lead to lower pull-out strength or more frequent graft incorporation complications [23]. Graft length inside the tunnels might therefore be of less importance than previously thought.

Regarding the fixation method, reports of graft failure after ACL reconstruction because of graft pull out or fixation failures are rare, suggesting that available graft fixation methods are adequate. Regarding strength of the graft, there are studies of the biomechanical properties of tendons that indicate that the four-strand semitendinosus tendon should provide a stiffer and stronger graft than the doubled semitendinosus and gracilis of the same diameter [1]. In theory a stiffer graft with larger graft diameter should result in fewer graft ruptures, but to our knowledge no results from large cohorts regarding graft rupture frequencies comparing ACL reconstructions with ST or ST/G have been published. In this study the difference in stiffness and the effect of a larger graft diameter in the ST group did not result in any clinically observable difference regarding rotational or translational laxity between the graft groups. In our view, a four strand semitendinosus ACL reconstruction is more technically demanding than a ST/G reconstruction, and aside from an attempt to minimise harm, no beneficial results from this study were found to advocate this more demanding procedure.

There are some limitations to this study. There is a risk of bias as this is a retrospective study of patients with few and minor additional injuries to their ACL rupture. However gender, age and additional injuries were evenly distributed between the groups. Measuring true rotational laxity in a setting similar to the pivoting demands posed in sports is difficult. A number of studies have nonetheless measured tibial rotation during pivoting using gait analysis techniques, and all report comparable values regarding rotational laxity $[12,13$, $20,24]$. The use of marker-based gait analysis to measure knee rotation motion may be subject to error from marker misplacement and artefact from skin movement. It has been shown that the error arising from marker misplacement is substantially reduced when the assessors are experienced [25]. All measurements in this study were performed by the same two assessors with over ten years of experience in gait analysis [25]. The good repeatability of data from these assessors has been reported by Webster et al. [24].

In conclusion, we did not find any effect of the gracilis tendon harvest on either the outcome or graft related morbidity after hamstring ACL reconstruction. The clinical relevance of our study is that we did not find evidence to warrant performing the more demanding ACL reconstruction with semitendinosus tendon only. 


\section{References}

1. Noyes FR, Butler DL, Grood ES, Zernicke RF, Hefzy MS (1984) Biomechanical analysis of human ligament grafts used in kneeligament repairs and reconstructions. J Bone Joint Surg [Am] 66 (3):344-352

2. Yunes M, Richmond JC, Engels EA, Pinczewski LA (2001) Patellar versus hamstring tendons in anterior cruciate ligament reconstruction: a meta-analysis. Arthroscopy 17(3):248-257

3. Barenius B, Nordlander M, Ponzer S, Tidermark J, Eriksson K (2010) Quality of life and clinical outcome after anterior cruciate ligament reconstruction using patellar tendon graft or quadrupled semitendinosus graft: an 8-year follow-up of a randomized controlled trial. Am J Sports Med 38(8):1533-1541

4. Pinczewski LA, Lyman J, Salmon LJ, Russell VJ, Roe J, Linklater J (2007) A 10-year comparison of anterior cruciate ligament reconstructions with hamstring tendon and patellar tendon autograft: a controlled, prospective trial. Am J Sports Med 35(4):564-574

5. Duquin TR, Wind WM, Fineberg MS, Smolinski RJ, Buyea CM (2009) Current trends in anterior cruciate ligament reconstruction. J Knee Surg 22(1):7-12

6. Nakamura N, Horibe S, Sasaki S, Kitaguchi T, Tagami M, Mitsuoka T, Toritsuka Y, Hamada M, Shino K (2002) Evaluation of active knee flexion and hamstring strength after anterior cruciate ligament reconstruction using hamstring tendons. Arthroscopy 18(6):598-602

7. Viola RW, Sterett WI, Newfield D, Steadman JR, Torry MR (2000) Internal and external tibial rotation strength after anterior cruciate ligament reconstruction using ipsilateral semitendinosus and gracilis tendon autografts. Am J Sports Med 28(4):552-555

8. Tashiro T, Kurosawa H, Kawakami A, Hikita A, Fukui N (2003) Influence of medial hamstring tendon harvest on knee flexor strength after anterior cruciate ligament reconstruction. A detailed evaluation with comparison of single-and double-tendon harvest. Am J Sports Med 31(4):522-529

9. Gobbi A (2010) Single versus double hamstring tendon harvest for ACL reconstruction. Sports Med Arthrosc 18(1):15-19

10. Ardern CL, Webster KE, Taylor NF, Feller JA (2010) Hamstring strength recovery after hamstring tendon harvest for anterior cruciate ligament reconstruction: a comparison between graft types. Arthroscopy 26(4):462-469

11. Segawa H, Omori G, Koga Y, Kameo T, Iida S, Tanaka M (2002) Rotational muscle strength of the limb after anterior cruciate ligament reconstruction using semitendinosus and gracilis tendon. Arthroscopy 18(2):177-182
12. Ristanis S, Stergiou N, Patras K, Tsepis E, Moraiti C, Georgoulis AD (2006) Follow-up evaluation 2 years after ACL reconstruction with bone-patellar tendon-bone graft shows that excessive tibial rotation persists. Clin J Sport Med 16(2):111-116

13. Georgoulis AD, Ristanis S, Chouliaras V, Moraiti C, Stergiou N (2007) Tibial rotation is not restored after ACL reconstruction with a hamstring graft. Clin Orthop Relat Res 454:89-94

14. Webster KE, Wittwer JE, O'Brien J, Feller JA (2005) Gait patterns after anterior cruciate ligament reconstruction are related to graft type. Am J Sports Med 33(2):247-254

15. Webster KE, Feller JA (2011) Alterations in joint kinematics during walking following hamstring and patellar tendon anterior cruciate ligament reconstruction surgery. Clin Biomech (Bristol, Avon) 26(2):175-180

16. Noyes FR, Stabler CL (1989) A system for grading articular cartilage lesions at arthroscopy. Am J Sports Med 17(4):505-513

17. Irrgang JJ, Anderson AF, Boland AL, Harner CD, Kurosaka M, Neyret P, Richmond JC, Shelborne KD (2001) Development and validation of the international knee documentation committee subjective knee form. Am J Sports Med 29(5):600-613

18. Davis Iii RB, Ounpuu S, Tyburski D, Gage JR (1991) A gait analysis data collection and reduction technique. Hum Mov Sci 10(5):575-587

19. Kadaba MP, Ramakrishnan HK, Wootten ME (1990) Measurement of lower extremity kinematics during level walking. J Orthop Res $8(3): 383-392$

20. Webster KE, Palazzolo SE, McClelland JA, Feller JA (2012) Tibial rotation during pivoting in anterior cruciate ligament reconstructed knees using a single bundle technique. Clin Biomech (Bristol, Avon) 26(5):480-484

21. Prodromos $C$ (2007) The anterior cruciate ligament reconstruction and basic science. $2 \mathrm{ST} / 2 \mathrm{Gr}, 4 \mathrm{ST}$, and $3 \mathrm{ST} / 2 \mathrm{Gr}$ techniques: deciding which hamstring configuration to use. Saunders, pp 110-114

22. Ma CB, Keifa E, Dunn W, Fu FH, Harner CD (2010) Can preoperative measures predict quadruple hamstring graft diameter? Knee 17(1):81-83

23. Zantop T, Ferretti M, Bell KM, Brucker PU, Gilbertson L, Fu FH (2008) Effect of tunnel-graft length on the biomechanics of anterior cruciate ligament-reconstructed knees: intra-articular study in a goat model. Am J Sports Med 36(11):2158-2166

24. Webster KE, McClelland JA, Wittwer JE, Tecklenburg K, Feller JA (2010) Three dimensional motion analysis of within and between day repeatability of tibial rotation during pivoting. Knee 17 (5):329-333

25. McGinley JL, Baker R, Wolfe R, Morris ME (2009) The reliability of three-dimensional kinematic gait measurements: a systematic review. Gait Posture 29(3):360-369 